\title{
Perception of BDS students of Kathmandu University on online learning during COVID-19 pandemic
}

\author{
Dr Alka Gupta', Dr Rabindra Man Shrestha², Dr Sujita Shrestha³, Dr Asal Acharya4, \\ Dr Nashib Pandey ${ }^{5}$ \\ 1,4Lecturer, ${ }^{2}$ Professor, Department of Orthodontics, ${ }^{3}$ Lecturer, Department of Public Health Dentistry, \\ ${ }^{5}$ Lecturer, Department of Periodontics, Kantipur Dental College, Kathmandu, Nepal \\ Corresponding author: Dr Alka Gupta; Email:alkagupta985@gmail.com
}

\section{ABSTRACT}

Introduction: The spread of COVID-19pandemic has gripped the entire world and caused widespread public health concerns, hampered economics and education system immensely. Online classes have been an alternative to give continuity to the theory classes. The objective of this study is to assess the various aspects of online classes and perception of Bachelor of Dental Surgery (BDS) students of Kathmandu University (KU) during COVID-19 pandemic.

Materials and Method: Cross-sectional, questionnaire based descriptive study using online Google form was distributed among the BDS students of KU. The questionnaire was distributed via social media. Frequency distribution of the descriptive data was done. Chi-square test was done to assess the difference in perception about online classes between the basic science and clinical science students.

Result: $89 \%$ of the students had never attended any online classes before online education due to COVID-19 pandemic. $76.9 \%$ agreed that the online class is distracting. $57.5 \%$ used smartphone for seeking online class and medium most frequently used was Zoom platform. $70.2 \%$ students could ask questions, communicate \& receive response during online class. $55.4 \%$ students disagreed that online classes are more effective. Statistical significant differences were seen in students submitting assignments, communication and practical simulation among basic science and clinical science students.

Conclusion: Online class can serve as an alternative effective educational tool. With more practice, system upgrading, capacity building of the student-teacher; it is bound to be more effective as well as efficient. The online class should be designed in such a way that student can focus and find it more interesting and should introduce various strategies to increase the interaction between students and teachers. Further, training on online class is required for both course recipients and providers.

KEYWORDS: BDS students, lockdown, online class, pandemic, perception.

\section{INTRODUCTION}

Corona virus disease 2019 (COVID-19) is an infectious disease caused by a novel virus which belongs to the virus family known as Corona virus. It has spread globally, resulting in Corona virus pandemic and gripped the entire world. World Health Organization (WHO) has declared COVID-19, a public health emergency and pandemic. Nepal, too is fighting COVID-19 pandemic like other countries in the world. Despite the continuous efforts to control the pandemic, COVID-19 cases have been persistently spreading and numbers are increasing day-by-day.

COVID-19 pandemic lockdown resulted in the closure of the vast majority of educational institutes like schools, colleges, universities etc worldwide. ${ }^{1,2}$ These nationwide closures are impacting over $60 \%$ of the world's student population. Several other countries have implemented localized closures impacting millions of additional learners. Online learning has become the alternative 
for education in order to control the risk of community transmission. Many educational institutes shifted to online learning via platforms like Zoom, Google Meet, Google Classroom, Microsoft Teams, D2L, Edgenuity etc. ${ }^{3,4}$

Digital technology can enable teachers and students to access specialized materials well beyond the textbooks, in multiple formats and in ways that can bridge time and space. The Organization for Economic Co-operation and Development has created framework to guide an education response to the COVID-19 pandemic for distance learning.

The aim of the study was to assess the perception of BDS students of KU on online learning during COVID-19 pandemic.

\section{MATERIALS AND METHOD}

The study design was descriptive, cross-sectional, questionnaire based which was conducted among the BDS students of Kathmandu University College namely; College of Medical Science (COMS), Kantipur Dental College (KDC), Kathmandu Medical College (KMC), Kathmandu University School of Medical Sciences (KUSMS), Nepal Medical College (NMC), and Nobel Medical College (NoMC). Structured questionnaire was used which consisted of two sections. The first section included demographic data and second section had questions about the perception of students on online learning during COVID-19 pandemic. The four likertscale ranged from "Strongly Agree", "Agree", "Disagree" to "Strongly Disagree". Further, five questions (Table 5) responses were merged to the category agreement i.e. (strongly agree \& agree) and category disagreement (disagree \& strongly disagree).

The study was conducted during May-July 2020. Ethical clearance was obtained from the IRC of Kantipur Dental College (IRC-16/020). BDS students studying under KU who were willing to participate, attending e-class and using social media were included. Sample size was calculated using the data from the study of Sub ediet $\mathrm{al}^{5}$ : Sample size $(\mathrm{n})=\mathrm{z}^{2} \mathrm{pq} / \mathrm{e}^{2}=708$.

Self administered online Google firm based survey questionnaire was sent via email, viber, facebook, etc to 1100 BDS students of KU. Among them, 894 students responded. Further, responses were refined and duplication was removed. Final analysis was done on 769 participants. Data was retrieved from the online survey, entered into Microsoft Excel and then imported into the Statistical Package for Social Sciences (SPSS) version 21. Frequency distribution was done for the descriptive data and Chi-square test was done to assess significant difference between the online class experience among the students. $p \leq 0.05$ was considered statistically significant.

\section{RESULT}

Table 1 highlights the demographic details of all respondents of $\mathrm{KU}$ dental colleges. Table 2 highlights the descriptive statistics on factual details of online class. Majority of the students used smartphone for seeking online class and medium used was Zoom platform. Table 3 highlights the dichotomous perceptional response of respondent on online class. $89.2 \%$ have never attended any online class before. $76.9 \%$ agreed that the online class is distracting. Table 4 shows the perceptional response of all respondents. $70.2 \%$ students can ask questions, communicate \& receive response during online class.

Table 5 highlights the level wise dichotomous perceptional differences on online class. Statistically significant differences were seen among the basic and clinical science students who were able to submit assignment, perform practical/ clinical simulation exercise through online classes and if training was required for faculty.

Table 6 highlights the College-wise factual differences on online class. Majority of the participants used Zoom platform and the modality of online class was via Microsoft PowerPoint presentation. Table 7 highlights the College-wise perceptional differences on online class. $66 \%$ were able to submit assignments and $47 \%$ disagreed to perform class test. $59 \%$ strongly disagreed that practical/ clinical simulation exercise could be conducted through online class. 
Table 1: Demographic details of all respondents of $\mathrm{KU}$ dental colleges

\begin{tabular}{|l|l|l|l|l|l|l|l|l|}
\hline \multicolumn{2}{|c|}{ Parameters } & COMS & KDCH & KMC & KUSMS & NMC & NoMC & Total \\
\hline \multirow{2}{*}{ Gender } & Male & 12 & 27 & 13 & 20 & 27 & 15 & $114(14.8 \%)$ \\
\cline { 2 - 9 } & Female & 46 & 151 & 104 & 135 & 166 & 53 & $655(85.2 \%)$ \\
\hline \multirow{3}{*}{$\begin{array}{c}\text { Level of } \\
\text { study }\end{array}$} & $\begin{array}{l}\text { Basic } \\
\text { Science }\end{array}$ & 26 & 99 & 68 & 80 & 114 & 21 & $408(53.1 \%)$ \\
\cline { 2 - 9 } & $\begin{array}{l}\text { Clinical } \\
\text { Science }\end{array}$ & 32 & 79 & 49 & 75 & 79 & 47 & $361(46.9 \%)$ \\
\hline Total & $58(7.5 \%)$ & $178(23.1 \%)$ & $117(15.2 \%)$ & $155(20.2 \%)$ & $193(25.1 \%)$ & $68(8.8 \%)$ & 769 \\
\hline
\end{tabular}

Note: Basic Science: $1^{\text {st }} 2^{\text {nd }}$ year; Clinical Science: $3^{\text {rd }-5^{\text {th }}}$ year

Table 2: Descriptive statistics on factual details of online class

\begin{tabular}{|l|l|l|l|l|}
\hline Questions & \multicolumn{4}{|c|}{ Responses } \\
\hline \multirow{2}{*}{$\begin{array}{l}\text { No of online classes attended per week } \\
\text { during COVID-19 lockdown }\end{array}$} & $1-6$ & $7-12$ & $13-18$ & $>18$ \\
\cline { 2 - 5 } & $173(22.5 \%)$ & $257(33.4 \%)$ & $289(37.6 \%)$ & $50(6.5 \%)$ \\
\hline \multirow{2}{*}{$\begin{array}{l}\text { No of hours spend/day using book/PC } \\
\text { for education other than online class }\end{array}$} & $<1$ & $1-3$ & $3-6$ & $>6$ \\
\cline { 2 - 5 } & $765(99.5 \%)$ & $4(0.5 \%)$ & - & - \\
\hline \multirow{2}{*}{$\begin{array}{l}\text { No of hours spend/day using social } \\
\text { site or TV for entertainment }\end{array}$} & $<1$ & $1-3$ & $3-6$ & $>6$ \\
\cline { 2 - 5 } & $43(5.6 \%)$ & $338(44 \%)$ & $264(34.3 \%)$ & $124(16.1 \%)$ \\
\hline \multirow{3}{*}{ Device used for internet } & Laptop & Desktop PC & Smartphone & Tablet \\
\cline { 2 - 5 } & $292(38 \%)$ & $2(0.3 \%)$ & $442(57.5 \%)$ & $33(4.3 \%)$ \\
\hline \multirow{3}{*}{ Connection for seeking online class } & Wifi & Landline internet & Cellular data & Others \\
\cline { 2 - 5 } & $623(81 \%)$ & $44(5.7 \%)$ & $102(13.3 \%)$ & - \\
\hline \multirow{2}{*}{ Medium used for online class } & Zoom & Viber & Skype & Others \\
\cline { 2 - 5 } & $663(86.2 \%)$ & $1(0.1 \%)$ & $1(0.1 \%)$ & $104(13.5 \%)$ \\
\hline Modality of online class & MS PowerPoint & MS word & Tutorial class & Other \\
\cline { 2 - 5 } & $710(92.3 \%)$ & $13(1.7 \%)$ & $21(2.7 \%)$ & $25(3.3 \%)$ \\
\hline \multirow{3}{*}{ Condition of internet connection } & Good & Satisfactory & Bad & $\begin{array}{l}\text { Disturbed } \\
\text { electricity }\end{array}$ \\
\cline { 2 - 5 } & $99(12.9 \%)$ & $313(40.7 \%)$ & $107(13.9 \%)$ & $250(32.5 \%)$ \\
\hline
\end{tabular}

Table 3: Dichotomous perceptional response of respondent on online class

\begin{tabular}{|l|l|l|}
\hline Questions & Yes & No \\
\hline Online classes attended before Covid-19 pandemic & $83(10.8 \%)$ & $686(89.2 \%)$ \\
\hline Able to access internet easily & $529(68.8)$ & $240(31.2)$ \\
\hline Internet class causing economic burden & $164(21.3 \%)$ & $605(78.7 \%)$ \\
\hline Online class environment is distracting & $591(76.9 \%)$ & $178(23.1 \%)$ \\
\hline
\end{tabular}


Table 4: Perceptional response of all respondents

\begin{tabular}{|l|l|l|l|l|}
\hline Questions & Strongly Agree & Agree & Disagree & Strongly Disagree \\
\hline Able to submit assignment & $78(10.1 \%)$ & $506(65.8 \%)$ & $158(20.5 \%)$ & $27(3.5 \%)$ \\
\hline $\begin{array}{l}\text { Can ask questions, communicate \& receive } \\
\text { response }\end{array}$ & $192(25 \%)$ & $540(70.2 \%)$ & $31(4 \%)$ & $6(0.8 \%)$ \\
\hline $\begin{array}{l}\text { Practical/ clinical simulation exercise } \\
\text { through online class }\end{array}$ & $8(1 \%)$ & $22(2.9 \%)$ & $286(37.2 \%)$ & $453(58.9 \%)$ \\
\hline Possible to perform class test & $12(1.6 \%)$ & $133(17.3 \%)$ & $358(46.6 \%)$ & $266(34.6 \%)$ \\
\hline Training required for faculty & $121(15.7 \%)$ & $481(62.5 \%)$ & $163(21.2 \%)$ & $4(0.5 \%)$ \\
\hline
\end{tabular}

Table 5: Level wise dichotomous perceptional differences on online class

\begin{tabular}{|l|l|l|l|l|}
\hline Questions & Response & $\begin{array}{l}\text { Basic Sciences } \\
(\mathrm{n}=408)\end{array}$ & $\begin{array}{l}\text { Clinical Sciences } \\
(\mathrm{n}=361)\end{array}$ & $\mathrm{p}$-value \\
\hline \multirow{3}{*}{ Able to submit assignment } & Agree & $323(55.3 \%)$ & $261(44.7 \%)$ & $0.026^{\star}$ \\
\cline { 2 - 5 } & Disagree & $85(45.9 \%)$ & $100(54.1 \%)$ & \\
\hline \multirow{2}{*}{$\begin{array}{l}\text { Can ask questions, communicate \& } \\
\text { receive response }\end{array}$} & Agree & $389(53.1 \%)$ & $343(46.9 \%)$ & 0.831 \\
\cline { 2 - 5 } & Disagree & $19(51.4 \%)$ & $18(48.6 \%)$ & \\
\hline \multirow{2}{*}{$\begin{array}{l}\text { Practical/ clinical simulation exercise } \\
\text { through online class. }\end{array}$} & Agree & $8(26.7 \%)$ & $22(73.3 \%)$ & $0.003^{\star}$ \\
\hline \multirow{2}{*}{ Possible to perform class test } & Disagree & $400(54.1 \%)$ & $339(45.9 \%)$ & \\
\hline \multirow{2}{*}{ Training required for faculty } & Agree & $69(47.6 \%)$ & $76(52.4 \%)$ & 0.143 \\
\hline & Disagree & $339(54.3 \%)$ & $285(45.7 \%)$ & \\
\hline & Agree & $306(50.8 \%)$ & $296(49.2 \%)$ & $0.019^{\star}$ \\
\cline { 2 - 5 } & Disagree & $102(61.1 \%)$ & $65(38.9 \%)$ & \\
\hline
\end{tabular}

*Statistically Significant

Table 6: College-wise factual differences on online class

\begin{tabular}{|l|l|l|l|l|l|l|l|l|}
\hline \multirow{3}{*}{ Question } & \multicolumn{7}{|c|}{ Name of the College } \\
\cline { 2 - 9 } & Distribution & COMS & KDCH & KMC & KUSMS & NMC & NoMC & Total \\
\cline { 2 - 9 } & & $\mathrm{n}=58$ & $\mathrm{n}=178$ & $\mathrm{n}=117$ & $\mathrm{n}=155$ & $\mathrm{n}=193$ & $\mathrm{n}=68$ & $\mathrm{n}=769$ \\
\hline \multirow{2}{*}{$\begin{array}{l}\text { No of } \\
\text { online } \\
\begin{array}{l}\text { classes } \\
\text { attended } \\
\text { /week }\end{array}\end{array}$} & $1-6$ & $19(32.8 \%)$ & $88(49.4 \%)$ & $24(20.5 \%)$ & $26(16.8 \%)$ & $13(6.7 \%)$ & $3(4.4 \%)$ & $173(22.5 \%)$ \\
\cline { 2 - 9 } & $13-18$ & $11(19 \%)$ & $50(28.1 \%)$ & $45(38.5 \%)$ & $62(40 \%)$ & $97(50.3 \%)$ & $24(35.3 \%)$ & $289(37.6 \%)$ \\
\cline { 2 - 9 } & $>18$ & $6(10.3 \%)$ & $17(9.6 \%)$ & $6(5.1 \%)$ & $5(3.2 \%)$ & $13(6.7 \%)$ & $3(4.4 \%)$ & $50(6.5 \%)$ \\
\hline
\end{tabular}




\begin{tabular}{|c|c|c|c|c|c|c|c|c|}
\hline \multirow{2}{*}{$\begin{array}{l}\text { No of } \\
\text { hours } \\
\text { spend / } \\
\text { day using } \\
\text { book/ } \\
\text { PC for } \\
\text { education } \\
\text { other than } \\
\text { online } \\
\text { class }\end{array}$} & $<1$ & $58(100 \%)$ & $174(97.8 \%)$ & $117(100 \%)$ & $155(100 \%)$ & 193(100\%) & $68(100 \%)$ & $765(99.5 \%)$ \\
\hline & $1-3$ & - & $4(2.2 \%)$ & - & - & - & - & $4(0.5 \%)$ \\
\hline \multirow{4}{*}{$\begin{array}{l}\text { No of } \\
\text { hours } \\
\text { spend / } \\
\text { day using } \\
\text { internet/ } \\
\text { TV for } \\
\text { entertain- } \\
\text { ment }\end{array}$} & $<1$ & $1(1.7 \%)$ & $8(4.5 \%)$ & $9(7.7 \%)$ & $9(5.8 \%)$ & $10(5.2 \%)$ & $6(8.8 \%)$ & $43(5.6 \%)$ \\
\hline & $1-3$ & $33(56.9 \%)$ & $83(46.6 \%)$ & $54(46.2 \%)$ & $58(37.4 \%)$ & $79(40.9 \%)$ & $31(45.6 \%)$ & $338(44.0 \%)$ \\
\hline & $3-6$ & $20(34.5 \%)$ & $56(31.5 \%)$ & $33(28.2 \%)$ & $63(40.6 \%)$ & 69(35.8\%) & $23(33.8 \%)$ & 264(34.4\%) \\
\hline & $>6$ & $4(6.9 \%)$ & $31(17.4 \%)$ & $21(17.9 \%)$ & $25(16.1 \%)$ & $35(18.1 \%)$ & $8(11.8 \%)$ & $124(16.1 \%)$ \\
\hline \multirow{4}{*}{$\begin{array}{l}\text { Device } \\
\text { used for } \\
\text { internet }\end{array}$} & Laptop & $20(34.5 \%)$ & $66(37.1 \%)$ & $46(39.3 \%)$ & $72(46.5 \%)$ & 73(37.8\%) & $15(22.1 \%)$ & $292(38.1 \%)$ \\
\hline & Desktop PC & - & - & $2(1.7 \%)$ & - & - & - & $2(0.3 \%)$ \\
\hline & Smartphone & $36(62.1 \%)$ & $105(59 \%)$ & $62(53 \%)$ & $79(51 \%)$ & $109(56.5 \%)$ & $51(75 \%)$ & $442(57.5 \%)$ \\
\hline & Tablet & $2(3.4 \%)$ & $7(3.9 \%)$ & $7(6 \%)$ & $4(2.6 \%)$ & $11(5.7 \%)$ & $2(2.9 \%)$ & $33(4.3 \%)$ \\
\hline \multirow{3}{*}{$\begin{array}{l}\text { Connection } \\
\text { for seek- } \\
\text { ing online } \\
\text { class }\end{array}$} & Wifi & $49(84.5 \%)$ & $147(82.6 \%)$ & 103(88\%) & $133(85.8 \%)$ & $151(78.2 \%)$ & $40(58.8 \%)$ & $623(81 \%)$ \\
\hline & $\begin{array}{l}\text { Landline } \\
\text { internet }\end{array}$ & $3(5.2 \%)$ & $12(6.7 \%)$ & $4(3.4 \%)$ & $9(5.8 \%)$ & $11(5.7 \%)$ & $5(7.4 \%)$ & $44(5.7 \%)$ \\
\hline & $\begin{array}{l}\text { Cellular } \\
\text { data }\end{array}$ & $6(10.3 \%)$ & $19(10.7 \%)$ & $10(8.5 \%)$ & $13(8.4 \%)$ & $31(16.1 \%)$ & 23(33.8\%) & $102(13.3 \%)$ \\
\hline \multirow{4}{*}{$\begin{array}{l}\text { Medium } \\
\text { used for } \\
\text { online } \\
\text { class }\end{array}$} & Zoom & $56(96.6 \%)$ & $178(100 \%)$ & $117(100 \%)$ & $154(99.4 \%)$ & $90(46.6 \%)$ & $68(100 \%)$ & $663(86.2 \%)$ \\
\hline & Viber & $1(1.7 \%)$ & - & - & - & - & - & $1(0.1)$ \\
\hline & Skype & - & - & - & $1(0.6 \%)$ & - & - & $1(0.1 \%)$ \\
\hline & Others & $1(1.7 \%)$ & - & - & - & $103(53.4 \%)$ & - & $104(13.5 \%)$ \\
\hline \multirow{4}{*}{$\begin{array}{l}\text { Modality } \\
\text { of online } \\
\text { class }\end{array}$} & $\begin{array}{l}\text { MS Power- } \\
\text { point }\end{array}$ & $45(77.6 \%)$ & 172(96.6\%) & 109(93.2\%) & $147(94.8 \%)$ & $173(89.6 \%)$ & $64(94.1 \%)$ & $710(92.3 \%)$ \\
\hline & MS word & $1(1.7 \%)$ & $2(1.1 \%)$ & $3(2.6 \%)$ & $1(0.6 \%)$ & $6(3.1 \%)$ & - & $13(1.7 \%)$ \\
\hline & $\begin{array}{l}\text { Tutorial } \\
\text { class }\end{array}$ & $5(8.6 \%)$ & $3(1.7 \%)$ & $5(4.3 \%)$ & $5(3.2 \%)$ & $1(0.5 \%)$ & $2(2.9 \%)$ & $21(2.7 \%)$ \\
\hline & Other & $7(12.1 \%)$ & $1(0.6 \%)$ & - & $2(1.3 \%)$ & $13(6.7 \%)$ & $2(2.9 \%)$ & $25(3.3 \%)$ \\
\hline \multirow{4}{*}{$\begin{array}{l}\text { Condition } \\
\text { of internet } \\
\text { connection }\end{array}$} & Good & $10(17.2 \%)$ & $28(15.7 \%)$ & $12(10.3 \%)$ & $18(11.6 \%)$ & $18(9.3 \%)$ & 13(19.1\%) & $99(12.9 \%)$ \\
\hline & Satisfactory & $27(46.6 \%)$ & $62(34.8 \%)$ & $43(36.8 \%)$ & $68(43.9 \%)$ & $94(48.7 \%)$ & $19(27.9 \%)$ & $313(40.7 \%)$ \\
\hline & Bad & $5(8.6 \%)$ & $25(14 \%)$ & 15(12.8.2\%) & $18(11.6 \%)$ & $30(15.5 \%)$ & $14(20.6 \%)$ & $107(13.9 \%)$ \\
\hline & $\begin{array}{l}\text { Disturbed } \\
\text { electricity }\end{array}$ & $16(27.6 \%)$ & $63(35.4 \%)$ & $47(40.2 \%)$ & $51(32.9 \%)$ & $51(26.4 \%)$ & $22(32.4 \%)$ & $250(32.5 \%)$ \\
\hline
\end{tabular}


Table 7: College-wise perceptional differences on online class

\begin{tabular}{|c|c|c|c|c|c|c|c|c|}
\hline \multirow{3}{*}{ Questions } & \multicolumn{8}{|c|}{ Name of the College } \\
\hline & \multirow{2}{*}{ Response } & COMS & $\mathrm{KDCH}$ & KMC & KUSMS & NMC & NoMC & Total \\
\hline & & $58(100 \%)$ & 178(100\%) & $117(100 \%)$ & 155(100\%) & 193(100\%) & $68(100 \%)$ & 769 \\
\hline \multirow{4}{*}{$\begin{array}{l}\text { Able to } \\
\text { submit } \\
\text { assignment. }\end{array}$} & $\begin{array}{l}\text { Strongly } \\
\text { Agree }\end{array}$ & $2(3.4 \%)$ & $5(2.8 \%)$ & $14(12 \%)$ & $25(16.1 \%)$ & $29(15 \%)$ & $3(4 \%)$ & $78(10 \%)$ \\
\hline & Agree & $25(43.1 \%)$ & $112(62.9 \%)$ & $87(74.4 \%)$ & $119(76.8 \%)$ & $137(71 \%)$ & 26 (38\%) & $506(66 \%)$ \\
\hline & Disagree & $26(44.8 \%)$ & $53(29.8 \%)$ & $13(11.1 \%)$ & $11(7.1 \%)$ & 24 (12\%) & $31(46 \%)$ & $158(21 \%)$ \\
\hline & $\begin{array}{l}\text { Strongly } \\
\text { Disagree }\end{array}$ & $5(8.6 \%)$ & $8(4.5 \%)$ & $3(2.6 \%)$ & - & $3(2 \%)$ & $8(12 \%)$ & $27(4 \%)$ \\
\hline \multirow{4}{*}{$\begin{array}{l}\text { Can ask } \\
\text { questions, } \\
\text { commu- } \\
\text { nicate \& } \\
\text { receive } \\
\text { response. }\end{array}$} & $\begin{array}{l}\text { Strongly } \\
\text { Agree }\end{array}$ & $11(19 \%)$ & $44(24.7 \%)$ & $37(31.6 \%)$ & $43(27.7 \%)$ & $42(22 \%)$ & $15(22 \%)$ & $192(25 \%)$ \\
\hline & Agree & $40(69 \%)$ & $127(71.3 \%)$ & 76 (65\%) & $110(71 \%)$ & 144 (75\%) & 43 (63\%) & $540(70 \%)$ \\
\hline & Disagree & $5(8.6 \%)$ & $7(3.9 \%)$ & $4(3.4 \%)$ & $2(1.3 \%)$ & $6(3 \%)$ & $7(10 \%)$ & $31(4 \%)$ \\
\hline & \begin{tabular}{|l} 
Strongly \\
Disagree
\end{tabular} & 2 (3.4\%) & - & - & - & $1(1 \%)$ & $3(4 \%)$ & $6(1 \%)$ \\
\hline \multirow{4}{*}{$\begin{array}{l}\text { Practical/ } \\
\text { clinical } \\
\text { simulation } \\
\text { exercise } \\
\text { through } \\
\text { online } \\
\text { class. }\end{array}$} & $\begin{array}{l}\text { Strongly } \\
\text { Agree }\end{array}$ & - & $2(1.1 \%)$ & $1(0.9 \%)$ & $3(1.9 \%)$ & $1(1 \%)$ & $1(1 \%)$ & $8(1 \%)$ \\
\hline & Agree & $1(1.7 \%)$ & $4(2.2 \%)$ & $7(6.0 \%)$ & $1(0.6 \%)$ & $6(3 \%)$ & $3(4 \%)$ & $22(3 \%)$ \\
\hline & Disagree & $27(46.6 \%)$ & 72(40.4\%) & $40(34.2 \%)$ & $59(38.1 \%)$ & $68(35 \%)$ & $20(29 \%)$ & $286(37 \%)$ \\
\hline & $\begin{array}{l}\text { Strongly } \\
\text { Disagree }\end{array}$ & $30(51.7 \%)$ & $100(56.2 \%)$ & $69(59 \%)$ & 92(59.4\%) & 118(61\%) & $44(65 \%)$ & $453(59 \%)$ \\
\hline \multirow{4}{*}{$\begin{array}{l}\text { Possible } \\
\text { to perform } \\
\text { class test. }\end{array}$} & $\begin{array}{l}\text { Strongly } \\
\text { Agree }\end{array}$ & $3(5.2 \%)$ & $1(0.6 \%)$ & $4(3.4 \%)$ & $1(0.6 \%)$ & $1(1 \%)$ & $2(3 \%)$ & $12(2 \%)$ \\
\hline & Agree & $16(27.6 \%)$ & 29(16.3\%) & 30 (25.6\%) & 19(12.3\%) & $32(17 \%)$ & $7(10 \%)$ & $133(17 \%)$ \\
\hline & Disagree & $23(49.7 \%)$ & $84(47.2 \%)$ & 47 (40.2\%) & 89 (57.4\%) & 90 (47\%) & $25(37 \%)$ & $358(47 \%)$ \\
\hline & $\begin{array}{l}\text { Strongly } \\
\text { Disagree }\end{array}$ & $16(27.6 \%)$ & $64(36 \%)$ & $36(30.8 \%)$ & $46(29.7 \%)$ & $70(36 \%)$ & $34(50 \%)$ & $266(35 \%)$ \\
\hline \multirow{4}{*}{$\begin{array}{l}\text { Training } \\
\text { required for } \\
\text { faculty. }\end{array}$} & $\begin{array}{l}\text { Strongly } \\
\text { Agree }\end{array}$ & $8(13.8 \%)$ & $30(16.9 \%)$ & $30(25.6 \%)$ & $15(9.7 \%)$ & $25(13 \%)$ & 13(19\%) & $121(16 \%)$ \\
\hline & Agree & $44(75.9 \%)$ & $112(62.9 \%)$ & 63(53.8\%) & $103(66.5 \%)$ & $115(60 \%)$ & $44(65 \%)$ & $481(63 \%)$ \\
\hline & Disagree & $6(10.3 \%)$ & $36(20.2 \%)$ & 23(19.7\%) & $36(23.3 \%)$ & $53(27 \%)$ & $9(13 \%)$ & $163(21 \%)$ \\
\hline & $\begin{array}{l}\text { Strongly } \\
\text { Disagree }\end{array}$ & - & - & $1(0.9 \%)$ & $1(0.60 \%)$ & - & $2(3 \%)$ & $4(1 \%)$ \\
\hline
\end{tabular}




\section{DISCUSSION}

In response to school closures caused by COVID-19, United Nations Educational, Scientific and Cultural Organization (UNESCO) recommended the use of distance learning program and open educational applications and platforms that schools and teachers can use to reach learners remotely and limit the disruption of education. ${ }^{6}$

Distance learning uses interactive radio instruction (IRI), interactive audio instruction (IAI), online virtual worlds, digital games, webinars, and webcasts, all of which are referred to as e-Learning. ${ }^{7}$ Upoalkpajor JN and Upoalkpajor CB revealed that COVID-19 pandemic has significant impact on education in Ghana. ${ }^{8}$

Among the total respondents, more than $2 / 3^{\text {rd }}$ of respondent never attended any online classes before. More than $1 / 3^{\text {rd }}$ of respondents attended $13-18$ classes/ day during COVID-19 lockdown. More than $2 / 3^{\text {rd }}$ of respondents were able to easily access internet as needed for their studies which was in accordance with Subedi et al. ${ }^{5}$ Majority respondents spend $<1$ hour/ day using book or PC for education purpose other than online classes. More than $1 / 3^{\text {rd }}$ of respondents spend 3-6 hours/day using internet, social site or TV for entertainment.

Poor internet connection was the major reason among the students who were not able to access the internet. Subedi et al concluded that $63.2 \%$ of students get disturbed for online class because of electricity problem. ${ }^{5}$ Eltahir, ${ }^{9}$ Esterhuyse and Scholtz, ${ }^{10}$ Islam et al, ${ }^{11}$ Al-Azawei et $\mathrm{al}^{12}{ }^{12}$ Nwabufo et $\mathrm{al}^{13}$ concluded that lack of technical support was one of the e-learning system failures. Unavailability of technical staff, lack of support of facilities to perform various activities, slow speed of internet and high internet traffic were encountered during online classes.

The students responded via laptop, desktop PC, smartphone and tablet. Majority of the respondents were comfortable and used smart phone for seeking online class which was in accordance with Subedi et al. ${ }^{5}$ They also stated that $56.1 \%$ have gadgets available at home for the online class. $40 \%$ used laptop/ computer. Parajuli KP concluded that mobile learning should be integrated in formal education system. ${ }^{14}$ Mobile phone dependence was common among the UG medical students which suggested the need to develop educational program to educate the students to use mobile phone meaningfully. ${ }^{15}$ Students were found to be using mobile phone excessively signaling the evolution of mobile phone use from a habit to an addiction. ${ }^{16}$

More than $2 / 3^{\text {rd }}$ of total respondents used wifi for seeking online classes. Subedi et al stated that $32.9 \%$ use internet data pack for their online classes while only $13.3 \%$ respondents used cellular data in our study. ${ }^{5}$

Different e-learning data packages has been launched by Nepalese telecom companies like Nepal Telecom and Ncell at an affordable rate regarding which most of the student are not aware of. More than $2 / 3^{\text {rd }}$ of total respondents experienced that internet class is not posing economic burden on them which could be due to the deduced cost of travelling, lodging and fooding. Although it seems feasible for majority of students from urban cities and well-to-do families, students from adverse families are affected. ${ }^{17}$ Students bear out of pocket expenditure for expensive data packages imposing added financial burden as ${ }^{13-18}$ classes were being taken per week during COVID-19 lockdown.

Internet connection experienced by the respondents was satisfactory for $1 / 3^{\text {rd }}$ of the total respondents. Subedi et al concluded that $63.6 \%$ of students get disturbed for online class because of internet problem whereas $63.2 \%$ gets disturbed for online class because of electricity problem. ${ }^{5}$ The geographical remoteness further hinders the network coverage across the country. ${ }^{17}$

The medium used for online classes was via Zoom, Viber, skype and others. More than $2 / 3^{\text {rd }}$ of total participants agreed that training is required for faculty/ instructor in order to conduct online classes. Out of the total respondents, $32.5 \%$ experienced weak internet due to disturbed electricity problem. Statistically significant differences were seen among the basic and clinical science students who were able to submit assignment, perform practical/ clinical simulation exercise through online classes and if training was required for faculty.

Parajuli $\mathrm{KP}^{14}$ concluded that mobile learning should be integrated in formal education system. Subedi et al ${ }^{5}$ concluded that e-learning is a good opportunity to continue education but in developing countries like 
Nepal it is not fully effective unless the factors affecting the E-learning process are taken into account. OwusuFordjour $\mathrm{C}$ et $\mathrm{al}^{18}$ recommended that students should be introduced to innovative and offline e-learning platforms to supplement classroom teaching.

More than half of total respondents disagreed that online classes are more effective which was in accordance with Owusu-Fordjour $\mathrm{C}$ et al. ${ }^{18} \mathrm{~J}$ Sandars, $S$ Schroter concluded that there is high awareness of new online technologies by both medical students and practitioners and high interest in its use for medical education. However, the potential of new technologies for UG and PG medical education can be achieved if there is increased training in how to use the new approach. ${ }^{19}$

COVID-19 pandemic have serious impacts on students' learning and well-being. Nepal has formulated a number of Information and communications technology (ICT) and education related policies since 2000; faulty implementation strategies and inability to implement those policies are the main challenges being experienced. ${ }^{20}$ Online teaching is feasible, cheap and must be made a part of the postgraduate training in India beyond the prevailing lockdown. ${ }^{21}$

\section{CONCLUSION}

Online class has been a reality since COVID-19 pandemic and served as an alternative educational tool. It paves a new pathway for mass as well as personalized education. With more practice, system upgrading, capacity building of the student-teacher; it is bound to be more effective as well as efficient. However, few significant issues encountered are transactional distance, lack of direct communication, domestic distraction, technological difficulties and practical barriers like poor internet connection and disturbed electricity problem and expensive data package for the students.

The online class should be designed in such a way that student can focus and find it more interesting and should introduce various strategies to increase the interaction between students and teachers. Further, training on online class is required for both recipients and course providers.

\section{REFERENCES}

1. COVID-19 Educational Disruption and Response. UNESCO. 4 March 2020. Retrieved 27 July 2020. Available from:https://en.unesco.org/ covid19/educationresponse

2. Reimers FM. Schleicher A. A framework to guide an education response to the COVID-19 Pandemic of 2020. OECD; 2020. Retrieved 5 July2020. Available from: https://oecd.dam-broadcast.com/pm_7379_126_126988-t63lxosohs.pdf

3. Micaela H. Pocono Record. Virtual learning gets mixed reviews from Pocono parents. 2020 May 1 [Retrieved 5 July 2020]. Available from: https://www.poconorecord.com/lifestyle/20200501/virtual-learning-gets-mixed-reviews-from-pocono-parents

4. Jonathon R. Georgia awards \$21 million in digital learning grants. 2020 April 28 [Retrieved 5 July 2020]. Available from:https:// www. 11 alive.com/article/news/health/coronavirus/georgia-school-districts-digital-learning-grants-atlanta/85-3a783e3c-2642-44e98fa6-090012604481

5. Subedi S, Nayaju S, Subedi S, Shah SK, Shah JM. Impact of E-learning during COVID-19 pandemic among nursing students and teachers of Nepal. Intl J of Sci Healthcare Res. 2020;5(3):68-76.

6. 290 million students out of school due to COVID-19: UNESCO releases first global numbers and mobilizes response. UNESCO. 4 March 2020. Retrieved 6 March 2020. Available from: https://en.unesco.org/news/290-million-students-out-school-due-covid-19-unescoreleases-first-global-numbers-and-mobilizes

7. Burns M. Distance education for Teacher Training: Modes, Models and Methods. Washington DC: Education Development Center Inc. Retrieved 2012-09-10.

8. Upoalkpajor JN, and Upoalkpajor CB. The Impact of COVID-19 on Education in Ghana. Asian J of Edu and Social Std. 2020;9(1):23-33.

9. Eltahir ME. E-learning in developing countries: Is it a panacea? A case study of Sudan. IEEE Access. 2019;7:97784-92.

10. Esterhuyse M, Scholtz B. Barriers to e-learning in a developing country. An explorativestudy. In Proceedings of the 9th IDIA conference. 2015;354-67.

11. Islam N, Beer M, Slack F. E-learning challenges faced by academics in higher education. J of Edu and Training Std. 2015;3(5):102-12 
12. Al-Azawei A, Parslow P,Lundqvist K. Barriers and opportunities of e-learning implementation in Iraq: A case of public universities. The International Review of Research in Open and Distributed Learning. 2016;17(5):126-46.

13. Nwabufo BN, Umoru TA,Olukotun JO. The challenges of e-learning in tertiary institutions in Nigeria. In International Conference the Future of Education Florence, 2013.June.

14. Parajuli KP. Mobile Learning Practice in Higher Education in Nepal. Open Praxis. 2016;8(1):41-54.

15. Thapa K, Lama S, Pokharel R, Sigdel R \& Rimal SP. Mobile Phone Dependence among Undergraduate Students of a Medical College of Eastern Nepal: A Descriptive Cross-sectional Study. J Nepal Med Assoc. 2020;58(224):234-9.

16. Thapa K, Pokharel R, Sigdel R \& Rimal SP. Pattern of Mobile Phone Use among Students of An Institution. J Nepal Med Assoc. 2018;56(209):522-6.

17. Nepal B, Atreya A. Online Medical Education in Nepal: Barking a Wrong Tree. J Lumbini Med Coll. 2020;8(1):113-4.

18. Owusu-Fordjour C, Koomson CK, Hanson D. The Impact of Covid-19 on learning-The perspective of the Ghanaian student.2020;7(3):88-101.

19. Sandars J, Schroter S. Web 2.0 technologies for undergraduate and postgraduate medical education: an online survey. Postgrad Med J. 2007;83(986):759-62.

20. Dawadi, S, Giri RA, Simkhada P. Impact of COVID-19 on the Education Sector in Nepal: Challenges and Coping Strategies. Sage Submissions. Preprint. https://doi.org/10.31124/advance.12344336.v12020:

21. Agarwal S, Kaushik JS. Student's Perception of Online Learning during COVID Pandemic. Indian J Pediatr. 2020;87(7):554. 